CARNETS OE Carnets de géographes

GÉOGRAPHES.

2| 2011

Espaces virtuels

\title{
Miami la cubaine ? Pouvoir et circulation dans une ville carrefour entre les Amériques
}

Violaine Jolivet

\section{(2) OpenEdition}

Journals

Édition électronique

URL : http://journals.openedition.org/cdg/2918

DOI : $10.4000 /$ cdg. 2918

ISSN : 2107-7266

Éditeur

UMR 245 - CESSMA

Référence électronique

Violaine Jolivet, « Miami la cubaine ? Pouvoir et circulation dans une ville carrefour entre les

Amériques », Carnets de géographes [En ligne], 2 | 2011, mis en ligne le 02 mars 2011, consulté le 07

mai 2019. URL : http://journals.openedition.org/cdg/2918; DOI : 10.4000/cdg.2918

Ce document a été généré automatiquement le 7 mai 2019.

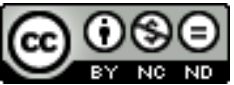

La revue Carnets de géographes est mise à disposition selon les termes de la Licence Creative Commons Attribution - Pas d'Utilisation Commerciale - Pas de Modification 4.0 International. 


\title{
Miami la cubaine ? Pouvoir et circulation dans une ville carrefour entre les Amériques
}

\author{
Violaine Jolivet
}

1 Cette thèse interroge les processus de construction d'une ville du sud des États-Unis (Miami et plus largement le comté de Miami-Dade) à travers le prisme de la relation spécifique qu'elle entretient avec une population d'immigrants qui revendique sa marque de fabrique sur ce carrefour entre les Amériques. Les Cubano-américains, qui ont majoritairement quitté l'île après la victoire de la révolution castriste (1959), composent aujourd'hui une part très importante de l'élite dirigeante de la cité (économique et surtout politique) et environ un tiers de ses habitants (cf. carte).

2 Ce sujet présente une étude approfondie de l'exil cubain (pour la majorité, la migration est comparable à un " one way ticket » avec un refus et/ou une impossibilité de retourner à Cuba à cause d'un embargo étatsunien et des lois migratoires cubaines), des conditions migratoires d'exception de cette population et de son accueil sur le sol étatsunien. Il invite à analyser la complexité des situations géopolitiques régionales d'hier (la révolution cubaine et la guerre froide) et d'aujourd'hui ainsi que les mutations politiques de Miami. L'articulation entre la géographie politique et une géographie plus sociale et culturelle est le point nodal de ce travail autour des relations entre l'espace et les sociétés à travers une approche attentive aux «enjeux de distinction, de marquage et d'appropriation de l'espace par certains groupes, certains pouvoirs » (Veschambre, 2008: 285).

3 La concentration à Miami des Cubains a fait émerger la cité comme le siège incontestable du contre-pouvoir cubain ; elle a permis une patrimonialisation et un marquage des lieux, en accord avec une autre histoire cubaine, loin des symboles révolutionnaires. Miami est désormais le cœur d'une seconde identité nationale définie par son articulation entre le territoire quitté et le territoire vécu. 


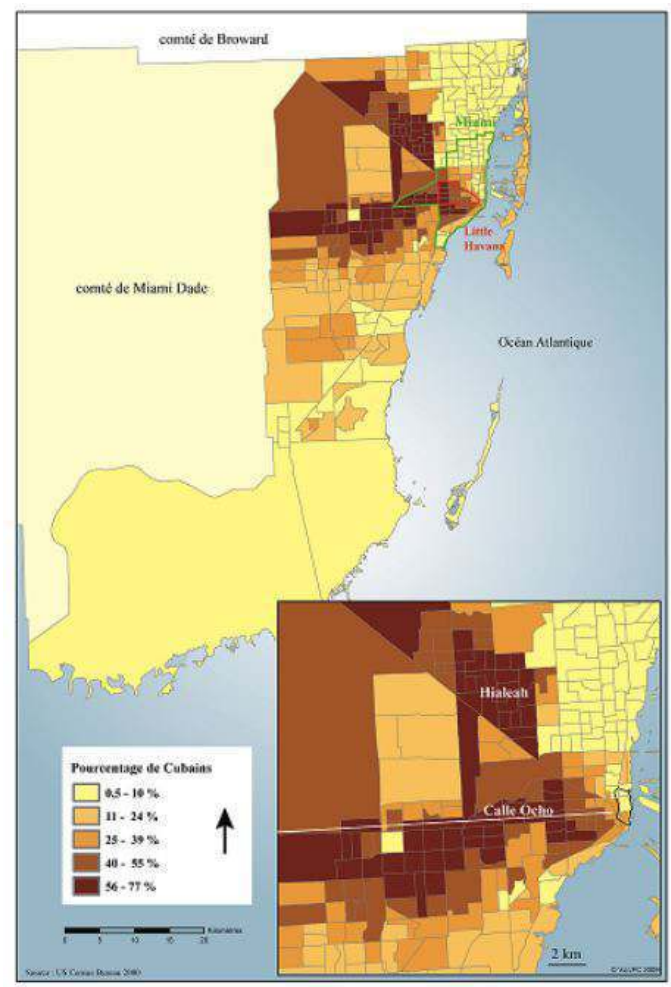

Ce territoire de l'entre-deux est le terreau de la puissance acquise par les Cubanoaméricains de Miami : ils ont pu et su développer de nouveaux réseaux économiques avec l'ensemble de l'Amérique et ouvrir résolument la ville aux investissements venus du Sud. Miami est aujourd'hui devenue un nœud des échanges intercontinentaux, se frayant une place de choix dans la hiérarchie des villes à l'échelle du globe. Elle a réussi à valoriser sa position stratégique, à l'heure de la mondialisation, pour devenir un véritable hub américain attirant les flux d'hommes et de capitaux.

Pourtant Miami est encore une ville en formation, une ville à bascule entre le Nord et le Sud selon nous. La jeunesse de la ville (Sunbelt city) et la diversité de ses habitants, si elles sont valorisées par un marketing urbain qui vante une ville cosmopolite, sont pourtant les vecteurs d'une ville fragmentée sans réelle centralité. L'appropriation spatiale de Miami par les Cubains, n'a pas changé fondamentalement les modes de constructions ainsi que « les logiques de l'exclusion » à l'échelle des quartiers (Elias, 1997) dans la ville. La production de territoires urbains reflète l'adaptation des Cubano-américains aux logiques néolibérales et étatsuniennes de production de la ville (privatisation, ségrégation et désengagement des pouvoirs publics) et compromet, dans cette ville pourtant dirigée par une minorité, un «droit à la ville » pour l'ensemble des citoyen-citadins (Lefebvre, 2000 a et $b)$.

6 Toutefois, depuis les années 1980, la ville de Miami s'est transformée sous l'impulsion des Cubains ainsi que par l'infusion des cultures cubaines, caribéennes et latines qui jouent sur les représentations comme les pratiques de la ville et font naître des nouvelles citadinités au cœur de ce carrefour. Ville du Nord, vécue par une majorité d'Hispaniques et dirigée par des Cubano-américains implantés depuis à peine un demi-siècle dans la cité, Miami est devenue une véritable plaque-tournante des échanges entre les mondes 
américains. Nouvel «Hollywood» latino-américain, la ville est aujourd'hui le lieu de production d'une "communauté hispanique » et de son paysage médiatique à l'échelle planétaire, montrant ainsi sa capacité à concentrer des moyens et des techniques qui concourent à sa renommée mondiale.

7 Ces nouvelles logiques spatiales font de Miami une ville en chantier, à la fois Babel et Babylone modernes, une ville centre et carrefour. Les interstices de ce chantier montrent cependant une créativité urbaine étonnante, qui inscrit Miami dans une dynamique relationnelle et communicationnelle entre les Amériques, symbole des créolisations inévitables (Glissant, 1997, 2000) auxquelles ce travail a porté une grande attention.

\section{Lien électronique vers la thèse \\ http://tel.archives-ouvertes.fr/tel-00558080/fr/ \\ Discipline \\ Géographie \\ Directeurs \\ J.L Chaléard, JM Théodat \\ Université \\ Université Paris 1 Panthéon Sorbonne}

Membres du jury de thèse, soutenue le 8 octobre 2010

Jean-Louis Chaléard, directeur (Université Paris 1 Panthéon Sorbonne)

Laurent Faret, (Université Paris-Diderot Paris 7)

Philippe Gervais-Lambony, (Université Paris-Ouest Nanterre La Défense)

Christian Girault, rapporteur, (CNRS)

Alex Stepick, rapporteur, (Professeur de sociologie à la Florida International University, Miami)

Jean Marie Théodat, directeur (Université Paris 1 Panthéon Sorbonne)

\section{Situation professionnelle actuelle}

Post-doctorat Fulbright au Center for Urban Research de la City University of New York

\section{BIBLIOGRAPHIE}

Elias N., 1997, Logiques de l'exclusion, Paris, Fayard

Glissant E., 1997, Traité du Tout-Monde, Paris, Gallimard

Glissant E., 2000, "La créolisation culturelle du monde" in Label France n ³8 pp. 1-3

Lefebvre H., 2000a [4 ${ }^{\text {ème }}$ ed], La production de l'espace, Paris, Anthropos 
Lefebvre H., 2000b [2 ${ }^{\text {ème }}$ ed], Espace et politique, Paris, Anthropos

Veschambre V., 2008, Traces et mémoires urbaines, Rennes, PUR

INDEX

Thèmes : Carnets de soutenances 